\title{
Mudanças de hábitos de vida em trabalhadores da atenção primária durante a pandemia de COVID-19: um estudo transversal
}

\author{
Changes in lifestyle habits in primary care workers during the Covid-19 \\ pandemic: a cross-sectional study \\ Cambios en los hábitos de vida de los trabajadores de atención primaria \\ durante la pandemia de Covid-19: un estudio transversal
}

\author{
Alberto SUMIYA ${ }^{(1)}$ \\ Eloisa PAVESI ${ }^{(1)}$ \\ Juliberta Alves de MACÊDO ${ }^{(2)}$ \\ Gabriel FARHAT ${ }^{(3)}$ \\ Celita Salmaso TRELHA ${ }^{(4)}$
}

Recebido: 4 nov. 2020

Revisado: 17 fev. 2021

Aceito: 23 fev. 2021

Autor de correspondência:

Alberto Sumiya

a.sumiya@ufsc.br

Conflito de interesses:

Os autores declaram não haver nenhum interesse profissional ou pessoal que possa gerar conflito de interesses em relação a este manuscrito.

\author{
(1)Universidade Federal de Santa Catarina - UFSC, Coordenadoria Especial de Saúde Única e \\ Biociências - BSU, Curso de Medicina, Curitibanos, SC, Brasil. \\ (2) Universidade Federal do Amazonas - UFAM, Instituto de Saúde e Biotecnologia - ISB, Curso de \\ Fisioterapia, Coari, AM, Brasil. \\ (3) Universidade Estadual de Ponta Grossa - UEPG, Centro de Ciências da Saúde - CCS, Ponta \\ Grossa, PR, Brasil. \\ (4)Universidade Estadual de Londrina - UEL, Departamento de Fisioterapia, Londrina, PR, Brasil.
}

\section{Resumo}

A pandemia de COVID-19 impôs um novo contexto social, permeado por muitas dificuldades e dúvidas, que levaram a sociedade a se adaptar ao chamado novo normal com mudanças de hábitos de vida, nem sempre saudáveis. Este estudo objetivou discutir algumas mudanças de hábitos de vida em trabalhadores da atenção primária em saúde provocadas pela pandemia de COVID-19 no Brasil. Para isso, foi realizada uma pesquisa transversal no período de 01/05/2020 a 31/05/2020. Utilizou-se um formulário online de 20 perguntas, que foi produzido no Google Forms ${ }^{\circledR}$ e disparado por e-mails, WhatsApp ${ }^{\circledR}$ e Facebook $^{\circledR}$. A amostra foi de conveniência e a análise dos dados foi descritiva pela verificação de frequências utilizando o Microsoft Excel ${ }^{\circledR}$ com alocação de respostas por Estado brasileiro, sendo o critério de inclusão respostas superiores a 30 por Estados. Os Estados incluídos foram Mato Grosso do Sul, Paraná e São Paulo, onde houve a predominância de participação do gênero feminino (89,1\%), que reportaram tempo de experiência de trabalho variando entre um a cinco anos (37,6\%). A maioria reportou medo moderado $(47,4 \%)$ de contrair o vírus e considerou que o pior ainda estava por vir $(67,5 \%)$ para o Brasil, sendo o aumento do sedentarismo $(65,8 \%)$ a principal mudança de hábito ocorrida. A pandemia evidenciou a modificação de hábitos de vida em trabalhadores da APS, principalmente em Fisioterapeutas e Enfermeiros, considerados profissionais da linha de frente. O maior impacto observado foi o sedentarismo, que pode levar ao desenvolvimento de doenças e afetar o trabalho.

Descritores: Infecções por Coronavírus; Pessoal de Saúde; Atenção Primária à Saúde; Hábitos. 


\section{Abstract}

The COVID-19 pandemic imposed a new social context, permeated by many difficulties and doubts, which led society to adapt to the so-called new normal with changes in lifestyle habits, not always healthy. This study aimed to discuss some lifestyle changes in primary health care workers caused by the COVID-19 pandemic in Brazil. For this, a cross-sectional research was carried out from 01/05/2020 to $05 / 31 / 2020$. An online form of 20 questions was used, which was produced on Google Forms ${ }^{\circledR}$ and triggered by e-mails, WhatsApp ${ }^{\circledR}$ and Facebook ${ }^{\circledR}$. The sample was of convenience and the data analysis was descriptive by checking frequencies using Microsoft Excel ${ }^{\circledR}$ with allocation of responses by Brazilian State, with the inclusion criterion being responses greater than 30 by States. The states included were Mato Grosso do Sul, Paraná and São Paulo, where there was a predominance of female participation (89.1\%), who reported time of work experience ranging from one to five years (37.6\%). Most reported moderate fear (47.4\%) of contracting the virus and considered that the worst was yet to come (67.5\%) for Brazil, with an increase in sedentary lifestyle (65.8\%) being the main change in habit occurred. The pandemic evidenced the change in life habits in PHC workers, mainly in Physiotherapists and Nurses, considered to be frontline professionals. The greatest impact observed was sedentary lifestyle, which can lead to the development of diseases and affect work.

Keywords: Coronavirus Infections; Health Personnel; Primary Health Care; Habits.

\section{Resumen}

La pandemia COVID-19 impuso un nuevo contexto social, impregnado de muchas dificultades y dudas, que llevaron a la sociedad a adaptarse a la llamada nueva normalidad con cambios en los hábitos de vida, no siempre saludables. Este estudio tuvo como objetivo discutir algunos cambios de estilo de vida en trabajadores de atención primaria de salud causados por la pandemia de COVID-19 en Brasil. Para ello, se realizó una investigación transversal desde el 05/01/2020 al 31/05/2020. Se utilizó un formulario en línea de 20 preguntas, que se produjo en Google Forms ${ }^{\circledR}$ y se activó por correo electrónico, WhatsApp ${ }^{\circledR}$ y Facebook ${ }^{\circledR}$. La muestra fue de conveniencia y el análisis de los datos fue descriptivo mediante la verificación de frecuencias en Microsoft Excel ${ }^{\circledR}$ con asignación de respuestas por Estado brasileño, siendo el criterio de inclusión las respuestas superiores a 30 por Estados. Los estados incluidos fueron Mato Grosso do Sul, Paraná y São Paulo, donde hubo un predominio de la participación femenina (89,1\%), quienes reportaron tiempo de experiencia laboral de uno a cinco años $(37,6 \%)$. La mayoría refirió temor moderado $(47,4 \%)$ de contraer el virus y consideró que lo peor estaba por venir $(67,5 \%)$ para Brasil, siendo el aumento del sedentarismo (65,8\%) el principal cambio de hábito ocurrido. La pandemia evidenció el cambio de hábitos de vida en los trabajadores de la APS, principalmente en Fisioterapeutas y Enfermeros, considerados profesionales de primera línea. El mayor impacto observado fue el sedentarismo, que puede conducir al desarrollo de enfermedades y afectar el trabajo.

Palabras-claves: Infecciones por Coronavirus; Personal de Salud; Atención Primaria de Salud; Hábitos.

\section{Introdução}

A nova Síndrome Respiratória Aguda por Coronavírus tipo 2 (SARS-CoV-2) identificado na China não é o primeiro coronavírus que teve disseminação rápida, antes vieram a Síndrome Respiratória Aguda por Coronavírus (SARS-CoV) em 2003, e depois a Síndrome Respiratória do Oriente Médio (MERS-CoV) em 2012, todos transmitidos por indivíduos pré-sintomáticos ou assintomáticos, que desafiam os sistemas de saúde do mundo. ${ }^{1}$

Diante dessa situação, no que se refere aos trabalhadores da saúde, manter a competência técnica e científica exige muito controle emocional, considerando que o cuidar apresenta riscos, responsabilidades e sofrimentos aumentados, podendo ocasionar 0 
adoecimento profissional, e assim impactar no processo de trabalho, e, consequentemente, ameaçar a qualidade e segurança dos serviços. ${ }^{2}$ De outra forma, o enfrentamento da COVID19, denunciou, inicialmente, muita angústia com a falta de treinamento rápido e informações convergentes, demora em propiciar infraestruturas adequadas e insumos suficientes.

Assim, continua sendo comum entre os profissionais da saúde o receio de infectar familiares, desenvolvendo nestes o sentimento de culpa e de impotência. Apesar disso, muitos são relutantes na busca de apoios, lançando mão de mecanismos de negação e racionalização para afastar as próprias fragilidades. Contudo, a intensificação dos desconfortos faz emergir sintomas, que levam, por exemplo, ao aumento do consumo de bebidas alcoólicas e a automedicação. ${ }^{3}$

Considerando-se experiências prévias de outros surtos e epidemias na literatura (Ebola, H1N1 e SARS), ${ }^{4}$ verificou-se que entre 11 e 73,4\% dos trabalhadores da saúde, principalmente médicos, enfermeiras e auxiliares tiveram sintomas que duraram até três anos, entre estes os sintomas depressivos (27,5 e 50,7\%), a insônia (34 e 36,1\%), ansiedade severa (45\%) e estresse $(18,1$ e $80,1 \%) .^{5}$

Desta forma, a hipótese deste artigo é que a COVID-19 provocou mudanças nos hábitos de vida dos trabalhadores da Atenção Primária à Saúde - APS devido a uma nova realidade social. A justificativa do estudo relaciona-se com a importância da proteção dessa força de trabalho, na medida em que ela representa a base fundamental do Sistema Único de Saúde - SUS, não somente no que concerne a promoção da saúde, mas neste momento, imprescindivelmente, o monitoramento e a vigilância.

Sendo assim, este estudo objetivou discutir as mudanças de hábitos de vida em trabalhadores da APS provocadas pela pandemia de COVID-19 no Brasil.

\section{Metodologia}

Trata-se de um estudo transversal, realizado entre 01/maio/2020 a 31/maio/2020 através de formulário online (Google Forms ${ }^{\circledR}$ ) cuja amostra de conveniência foi definida como sendo de profissionais de saúde atuantes na APS durante a pandemia de COVID-1 no Brasil. Os estudos transversais focam em populações bem definidas, sendo a observação mensurada uma única vez - um corte instantâneo de um fenômeno- como em inquéritos populacionais que utilizam dados primários. Em análises descritivas, os estudos transversais são amplamente utilizados para fomentar políticas públicas pelo reconhecimento de grupos vulneráveis, prevalência de exposições de risco e/ou vigilância de risco. São considerados baratos, simples e rápidos, não demandando seguimentos. ${ }^{6}$ 
O formulário teve como modelos de referência para sua construção websurveys de outros países como Canadá e Inglaterra. ${ }^{7,8} \mathrm{O}$ critério de inclusão para a análise dos dados foi número de respostas que deveria ser superior a 30 por Estado. Para a análise descritiva, buscando-se as frequências, utilizou-se o Microsoft Excel ${ }^{\circledR}$ para porcentagens e desvio padrão. Foram eliminadas as respostas incompletas e aqueles que não eram profissionais de saúde.

Assim, foram elaboradas 20 perguntas, sendo as seis primeiras de caráter sociodemográfico, as outras eram sobre o nível de preparado e de conhecimento, as necessidades de capacitação, as medidas adotadas, as mudanças de hábitos e comportamentos frente à COVID-19 (https://forms.gle/hffjnzAsSUjZqzoq5). O formulário foi disparado por meio de e-mails, WhatsApp ${ }^{\circledR}$ e Facebook ${ }^{\circledR}$. O tempo de preenchimento do mesmo foi de aproximadamente cinco minutos e durante a coleta de dados não houve nenhuma forma de registro da identidade dos sujeitos, o que garantiu anonimato, privacidade e sigilo.

Foram utilizadas três perguntas para a análise neste artigo: 1) você tem medo de contrair a COVID-19?; 2) Em geral, o que mudou na sua vida? 3) Na sua opinião, qual afirmação abaixo melhor descreve a situação do Brasil atualmente? Ademais, o estudo foi submetido ao Comitê de Ética em Pesquisa - CEP da Universidade Federal de Santa Catarina - UFSC e aprovado sob o parecer de número 4.049.849, de acordo com a Resolução n. 466/2012.

\section{Resultados}

Os estados brasileiros que conseguiram atingir mais de 30 respostas foram Mato Grosso do Sul (MS) ( $n=50)$, Paraná (PR) $(n=86)$ e São Paulo (SP) $(n=48)$, sendo que houve predominância do sexo feminino ( $M S=82,0 \%, P R=93,0 \%, S P=89,5 \%)$. As profissões mais frequentes foram Enfermagem no MS (18\%) e Fisioterapia no PR (45,3\%) e SP $(35,4 \%)$, onde o nível de escolaridade mais alta foi a de especialista (MS $=46,0 \%, P R=58,1 \%, S P=66,6 \%$ ), com tempo de trabalho na APS variando entre um a cinco anos (MS $=36,0 \%, P R=39,5 \%$, $\mathrm{SP}=35,4 \%)$ (Tabela 1).

Verificou-se que a maioria dos profissionais reportou medo moderado $(47,4 \%)$ de contrair a COVID-19, seguido por muito medo (27,3\%). Demonstrou-se prevalência quanto ao pessimismo em relação ao enfrentamento da COVID-19 no Brasil, tendo em vista as respostas o pior estava por vir $(67,5 \%)$ e estamos no pior período $(20,2 \%)$. 
ISSN 2179-6750

Tabela 1. Dados socioeconômicos e demográficos dos profissionais de saúde da APS

\begin{tabular}{|c|c|c|c|c|}
\hline \multirow[t]{2}{*}{ Variáveis } & MS $(n=50)$ & $P R(n=86)$ & $S P(n=48)$ & Total \\
\hline & $\mathrm{n}(\%)$ & n (\%) & n (\%) & n (\%) \\
\hline Idade média e desvio padrão & $37,8( \pm 8,4)$ & $39,3( \pm 8,3)$ & $40,1( \pm 8,2)$ & - \\
\hline \multicolumn{5}{|l|}{ Sexo } \\
\hline Feminino & $41(82,0)$ & $80(93,0)$ & $43(89,5)$ & $164(89,1)$ \\
\hline Masculino & $9(18,0)$ & $6(6,9)$ & $5(10,4)$ & $20(10,9)$ \\
\hline \multicolumn{5}{|l|}{ Escolaridade } \\
\hline Especialização & $23(46,0)$ & $50(58,1)$ & $32(66,6)$ & $105(57,4)$ \\
\hline Graduação & $18(36,0)$ & $8(9,3)$ & $7(14,5)$ & $33(18,1)$ \\
\hline Mestrado & $3(6,0)$ & $15(17,4)$ & $5(10,4)$ & $23(12,5)$ \\
\hline Ensino médio & $6(12,0)$ & $8(9,3)$ & $1(2,0)$ & $15(8,2)$ \\
\hline Doutorado & - & $5(5,8)$ & $2(4,1)$ & $7(3,8)$ \\
\hline \multicolumn{5}{|l|}{ Profissão } \\
\hline Fisioterapeuta & $1(2,0)$ & $39(45,3)$ & $17(35,4)$ & $57(31,6)$ \\
\hline Enfermeiro (a) & $9(18,0)$ & $10(11,6)$ & $4(8,3)$ & $23(12,4)$ \\
\hline Cirurgião-Dentista & $7(14,0)$ & $4(4,6)$ & $4(8,3)$ & $15(8,3)$ \\
\hline Agente Comunitário de Saúde & $3(6,0)$ & $8(9,3)$ & - & $11(6,1)$ \\
\hline Psicólogo & $3(6,0)$ & $2(2,3)$ & $6(12,5)$ & $11(6,1)$ \\
\hline Técnico Enfermagem & $6(12,0)$ & $3(3,4)$ & $1(2,0)$ & $10(5,6)$ \\
\hline Nutricionista & $4(8,0)$ & $3(3,4)$ & $3(6,2)$ & $10(5,6)$ \\
\hline Assistente Social & $6(12,0)$ & $1(1,1)$ & $2(4,1)$ & $9(4,9)$ \\
\hline Farmacêutico & $3(6,0)$ & $4(4,6)$ & - & $7(3,8)$ \\
\hline Fonoaudiólogo & $2(4,0)$ & $2(2,3)$ & $3(6,2)$ & $7(3,8)$ \\
\hline Médico & $3(6,0)$ & - & $3(6,2)$ & $6(3,3)$ \\
\hline Auxiliar de Enfermagem & - & $4(4,6)$ & - & $4(2,2)$ \\
\hline Educador Físico & - & $3(3,4)$ & - & $3(1,6)$ \\
\hline Terapeuta Ocupacional & - & $2(2,3)$ & - & $2(1,1)$ \\
\hline Auxiliar de Saúde Bucal & $2(4,0)$ & - & - & $2(1,1)$ \\
\hline Apoiador de Saúde & - & - & $2(4,1)$ & $2(1,1)$ \\
\hline Agente de Combate a Endemias & $1(2,0)$ & - & - & $1(0,5)$ \\
\hline Médico Veterinário & - & $1(1,1)$ & - & $1(0,5)$ \\
\hline Biólogo & - & - & $1(2,0)$ & $1(0,5)$ \\
\hline \multicolumn{5}{|l|}{ Tempo de trabalho } \\
\hline 01 a 05 anos & $18(36,0)$ & $34(39,5)$ & $17(35,4)$ & $69(37,6)$ \\
\hline 06 a 10 anos & $10(20,0)$ & $19(22,0)$ & $11(22,9)$ & $40(21,7)$ \\
\hline 11 a 15 anos & $8(16,0)$ & $13(15,1)$ & $3(6,2)$ & $24(13,1)$ \\
\hline Mais de 20 anos & $3(6,0)$ & $9(10,4)$ & $8(16,6)$ & $20(10,8)$ \\
\hline 01 a 12 meses & $7(14,0)$ & $4(4,6)$ & $5(10,4)$ & $16(8,7)$ \\
\hline 16 a 20 anos & $4(8,0)$ & $7(8,1)$ & $4(8,3)$ & $15(8,1)$ \\
\hline
\end{tabular}

Fonte: Elaborado pelos autores (2020).

No que concerne as mudanças, o sedentarismo (65,8\%) foi apontado como a principal mudança, depois vieram na sequência, o aumento do consumo de alimentos $(53,8 \%)$ e 
bebidas alcoólicas (17,4\%). Alguns profissionais reportaram ainda aumento de transtornos mentais $(12,5 \%)$ (Tabela 2).

Tabela 2. Grau de medo, situação do Brasil e mudanças de hábitos e comportamentos

\begin{tabular}{lcccc}
\hline \multicolumn{1}{c}{ Variáveis } & MS (n=50) & $\mathrm{PR}(\mathrm{n}=86)$ & $\mathrm{SP}(\mathrm{n}=48)$ & Total \\
\cline { 2 - 5 } & $\mathrm{n}(\%)$ & $\mathrm{n}(\%)$ & $\mathrm{n}(\%)$ & $\mathrm{n}(\%)$ \\
\hline Grau de medo & & & \\
Medo moderado & $23(46)$ & $37(43)$ & $27(56,5)$ & $87(47,4)$ \\
Muito medo & $15(30)$ & $30(34,9)$ & $10(21)$ & $50(27,3)$ \\
Pouco medo & $8(16)$ & $15(17,4)$ & $8(16,6)$ & $31(17)$ \\
Sem medo & $4(8)$ & $3(3,5)$ & $2(4,5)$ & $9(4,9)$ \\
Não sei dizer & 0 & $1(1,2)$ & $1(1,2)$ & $2(3,4)$ \\
Situação do Brasil & & & & \\
O pior está por vir & $32(64)$ & $57(66,3)$ & $35(73)$ & $124(67,5)$ \\
Estamos no pior período & $13(26)$ & $13(15,3)$ & $11(23)$ & $37(20,2)$ \\
Eu não sei dizer & $4(8)$ & $14(16,3)$ & $2(4)$ & $20(10,9)$ \\
O pior já passou & $1(2)$ & $2(2,1)$ & 0 & $3(1,4)$ \\
Mudanças de hábitos e comportamentos* & & & & \\
Sedentarismo & $27(54)$ & $61(71)$ & $33(68,8)$ & $121(65,8)$ \\
Consumo de alimentos & $29(58)$ & $42(48,9)$ & $28(58,4)$ & $99(53,8)$ \\
Consumo de bebidas alcoólicas & $7(14)$ & $14(16,3)$ & $11(23)$ & $32(17,4)$ \\
Consumo de medicamentos & $5(10)$ & $9(10,5)$ & $7(14,6)$ & $21(11,5)$ \\
Transtornos mentais & $4(8)$ & $13(15,2)$ & $6(12,5)$ & $23(12,5)$ \\
Outros & $2(4)$ & $8(9,4)$ & 0 & $10(5,5)$ \\
Tabagismo & $1(2)$ & $3(3,6)$ & 0 & $4(2,2)$ \\
\hline \hline
\end{tabular}

Fonte: Elaborado pelos autores (2020).

Nota: *Questão que permitia mais de uma resposta.

\section{Discussão}

Os profissionais de saúde constituem um grupo de categorias distintas, que atualmente estão diretamente implicados no atendimento às pessoas infectadas pela COVID-19 e, por este motivo, sujeitos aos riscos e ao adoecimento. São numerosos os desafios impostos, como por exemplo, as incertezas sobre a magnitude da pandemia (duração e efeitos da crise), as preocupações com o nível de preparo e conhecimento (curso da doença, lacunas nos protocolos de tratamento e falta de vacina), além do suprimento de material de proteção. ${ }^{9}$

Acrescentam a esse escopo, os longos períodos de trabalho, ${ }^{10}$ o estresse e o medo, que levam o trabalhador ao autoisolamento e a mudança de rotinas. Outro agravante é a relutância ou até mesmo a recusa, em aceitar suporte médico/terapêutico. ${ }^{11}$ Começam assim, a surgir sintomas subclínicos como dores de cabeça, desconfortos musculares, 
irritabilidade, hipervigilância, fadiga e distúrbios do sono, dentre outros. Estes, consequentemente, podem se agravar e transformar-se em desordens psiquiátricas como ansiedade e depressão. Isto posto, o desempenho laboral pode ser prejudicado por atitudes negativas, desengajamento, atrasos nos tratamentos, falhas de comunicação e absenteísmo. $^{12}$

Evidenciou-se que a maioria dos profissionais reportaram sentir medo moderado $(47,4 \%)$ e depois muito medo (27,3\%) de contrair a COVID-19. Uma pesquisa conduzida em hospitais públicos da cidade do Rio de Janeiro identificou elevada taxa de infecção pelo novo coronavírus entre os profissionais de saúde (25\%), muito acima daquelas verificadas na China (4\%) e Itália (15\%), em estágios mais avançados da pandemia. ${ }^{13}$ Além disso, em 6 de julho de 2020, registrava-se 176 óbitos de profissionais de saúde, com maior prevalência entre técnicos e auxiliares de enfermagem, seguidos por médicos e enfermeiros. ${ }^{14}$

Zerbini et al. ${ }^{9}$ complementam, apontando em pesquisa, que o medo de ser infectado pela COVID-19 se correlacionou com os escores aumentados do Malasch Burnout Inventory - MBI. Albott et al. ${ }^{11}$ verificou também que a exposição a seis ou mais fatores estressores ou a combinação de três específicos (execução de obrigações distante das habilidades, morte, doença grave de colega), representaram um alto risco de desenvolvimento de estresse póstraumático após apenas três meses do evento desencadeador. $\mathrm{E}$, que os piores resultados psicológicos foram encontrados em profissionais mais jovens, do gênero feminino, principalmente da enfermagem e aqueles com pobre saúde física.

Verificou-se também em nosso estudo a percepção negativa dos participantes sobre a situação do Brasil no enfrentamento da COVID-19. Interpretou-se este dado a partir da compreensão da exposição à mídia e às informações não sistematizadas ou desencontradas na época, e que exerceram influência na valoração de como a crise estava sendo gerenciada, determinando baixo nível de confiança diante da incapacidade de lideranças. ${ }^{15}$ Em alguma medida, o modelo capitalista na conjuntura da pandemia, revelou a pressão sobre o trabalho, com sua flexibilização (uberização), intensificação (mais horas e turnos) e precarização (falta de condições), que desregulam as relações sociais, desumanizam e descartam os menos adaptados (incompatibilidades entre os ritmos naturais e laborais). Produz-se um aumento da exploração, do estresse, da apreensão pelo medo do desemprego, cansaços e transtornos de saúde. ${ }^{16}$ Por isso, é importante a confiança, transparência e comunicação assertiva como elementos críticos no controle de epidemias, porque na presença de inconsistências, a população costuma refutar a possibilidade de sacrifícios. $^{17}$

Como percebido, mudanças de hábitos e comportamentos são esperados em situações de restrição e isolamento, porém algumas não repercutem adequadamente, 
afetando a autoeficácia. ${ }^{18}$ Observa-se uma tendência ao sedentarismo, ganho de peso e surgimento de comorbidades como obesidade, aumento da pressão arterial e intolerância a glicose, aumentando o risco cardiovascular. ${ }^{19}$ Situação preocupante porque pacientes crônicos são tidos como suscetíveis à COVID-19, independente da faixa etária. Desta forma, é consenso que a prática de exercício físico regular deve continuar, mesmo em reclusão domiciliar, como uma medida preventiva. ${ }^{19,20}$

Em função do isolamento e distanciamento social, deve-se realizar atividades físicas em casa e/ou ao ar livre sem aglomerações. Geralmente, a intensidade recomendada é moderada para se manter respostas positivas do sistema imunológico, a capacidade funcional e saúde mental. A frequência e duração podem variar de 30min diários e moderados, ou 20min em alta intensidade, porém a cada dois dias. Amplamente, 150min de exercícios aeróbicos por semana, que incluam exercícios de fortalecimento e flexibilidade. Os exercícios são assim, uma estratégia não-farmacológica para prevenção de doenças, metabólicas, físicas ou psicológicas. ${ }^{21}$

E, diretamente interligada a inatividade física está a questão nutricional, porque aumentam-se o número de refeições com a permanência prolongada em casa, o que não significa qualidade alimentar, já que o acesso a itens frescos é restringido pelo isolamento social, a renda familiar pode ter sido reduzida pelo desemprego e a mídia estimula o consumo de fastfood por meio de deliveries. 22,23

Os profissionais consultados neste artigo, relataram aumento do consumo de bebidas alcoólicas na ordem de $17,4 \%$, considerado um valor baixo se comparado com outra pesquisa que indicou $53,7 \%$. Sabe-se que o uso abusivo de álcool é o terceiro motivo de ausência do trabalho e que interfere na saúde e na qualidade de vida. Por apresentar capacidade ansiolítica, é obviamente mais consumido em situações limítrofes como mecanismo de fuga, ameniza sintomas, entretanto, como muitas drogas, pode levar a dependência. ${ }^{24}$

O aumento tabagismo foi outro problema levantado nesta pesquisa com os profissionais de saúde. A literatura evidencia que fumantes expostos a desastres consomem mais cigarros do que os não expostos e podem colocar os não fumantes em contato com emissões químicas, ${ }^{25}$ sendo maiores as chances de recaídas para aqueles que haviam cessado o vício. ${ }^{26}$ Vale salientar, portanto, que o tabagismo é fator de risco frente aos comprometimentos respiratórios causados pela COVID-19.

Assim, para continuar tendo motivação com o trabalho é preciso despertar a resiliência no profissional de saúde, instrumentalizando-o com habilidades para resistir as rupturas da normalidade com adaptação saudável e inteligência emocional. ${ }^{27} \mathrm{~A}$ resiliência permite enfrentar experiências de adversidade e sair fortalecido ou transformado. ${ }^{28}$ Para 
isso, é necessário então propiciar a criação de estruturas de assistência com estratégias de proteção e promoção da saúde física e mental do trabalhador, são exemplos: programas de educação em saúde com treinamentos, supervisão técnica com planos de contingenciamento, acompanhamento/monitoramento através de aplicativos eletrônicos e contato telefônico (hotlines e telehealth), instauração de grupos/redes de apoio, oferecimento de tratamento presencial, reforço dos programas de alimentação saudável e exercícios físicos ${ }^{11,12}$

À guisa de finalização desta discussão, compreende-se que é urgente e crítico trazer à tona para os gestores e autoridades responsáveis em saúde as dificuldades trazidas pela pandemia de COVID-19 aos trabalhadores desse setor.

\section{Conclusão}

O período de coleta de dados desta pesquisa coincidiu com o avanço da COVID-19 pelo Brasil, portanto os resultados expressaram um cenário inicial de aquisição de conhecimentos e inseguranças. A pandemia evidenciou a modificação de hábitos de vida em trabalhadores da APS, principalmente em Fisioterapeutas e Enfermeiros, considerados profissionais da linha de frente. O maior impacto observado foi o sedentarismo, que pode levar ao desenvolvimento de doenças e afetar o trabalho. Como medida, verifica-se a necessidade de maior valorização da Política Nacional de Saúde do Trabalhador e da Trabalhadora, para contextos pandêmicos. Por fim, a limitação do estudo foi a pesquisa ter sido online, a qual não teve o alcance territorial esperado, impedindo a análise de outros Estados brasileiros.

\section{Referências}

1. Pablo GS, Vaquerizo-Serrano J, Catalan A, Arango C, Moreno C, Ferre $C$, et al. Impact of coronavirus syndromes on physical and mental health of health care workers: Systematic review and meta-analysis. J Affect Disord. 2020 Oct 1;275:48-57.

https://doi.org/10.1016/j.jad.2020.06.022.

2. Dal'Bosco EB, Floriano LSM, Skupien SV, Arcaro G, Martins AR, Anselmo ACC. A saúde mental da enfermagem no enfrentamento da COVID-19 em um hospital universitário regional. Rev Bras Enferm. 2020;73(Suppl 2):e20200434. https://doi.org/10.1590/00347167-2020-0434. 
3. Braquehais MD, Vargas-Cáceres S, Gómez-Durán E, Nieva G, Valero S, Casas M, et al. The impact of the COVID-19 pandemic on the mental health of healthcare professionals. QJM. 2020 Jun 22;hcaa207. https://doi.org/10.1093/qjmed/hcaa207

4. Alwashmi MF. The use of digital health in the detection and management of COVID -19. Int J Environ Res Public Health. 2020 Apr 23;17(8):2906.

https://doi.org/10.3390/ijerph17082906.

5. Preti E, Di Mattei V, Perego G, Ferrari F, Mazzetti M, Taranto P, et al. The psychological impact of epidemic and pandemic outbreaks on healthcare workers: rapid review of the eidence. Curr Psychiatry Rep. 2020 Jul 10;22(8):43. https://doi.org/10.1007/s11920-02001166-z.

6. Sousa VD, Driessnack M, Mendes IAC. Revisão dos desenhos de pesquisa relevantes para enfermagem - parte 1: desenhos de pesquisa quantitativa. Rev Lat Am Enfermagem. 2007;15(3):502-7. https://doi.org/10.1590/S0104-11692007000300022.

7. Statistics Canada. COVID-19: a data perspective [Internet]. [local desconhecido]: Statistics Canada; 2020 [citado 3 abr. 2020]. Disponível em:

https://www150.statcan.gc.ca/n1/en/type/data?subject_levels=45\%2C4510

8. Office for National Statistics (UK). Coronavirus (COVID-19) [Internet]. [London]: ONS; 2020 [citado 3 abr. 2020]. Disponível em:

https://www.ons.gov.uk/peoplepopulationandcommunity/healthandsocialcare/conditionsa nddiseases/datalist?filter $=$ datasets

9. Zerbini G, Ebigbo A, Reicherts P, Kunz M, Messman H. Psychosocial burden of healthcare professionals in times of covid-19 - a survey conducted at the university hospital Augsburg. Ger Med Sci. 2020 Jun 22;18:Doc05. https://doi.org/10.3205/000281.

10. Silva LS, Machado EL, Oliveira HN, Ribeiro AP. Working conditions and lack of information on the impact of COVID-19 among health workers. Rev Bras Saude Ocup. 2020;45:e24. https://doi.org/10.1590/2317-6369000014520.

11. Albott CS, Wozniak JR, McGlinch BP, Wall MH, Gold BS, Vinogradov S. Battle buddies: rapid deployment of a psychological resilience intervention for health care workers during the Coranavirus disease pandemic. Anesth Analg. 2020;131(1):43-54. https://doi.org/ 10.1213/ANE.0000000000004912. 
12. Ornell F, Halpern SC, Kessler FHP, Narvaez JCM. The impact of the COVID-19 pandemic on the mental health of healthcare professionals. Cad Saude Publica. 2020 Apr 30;36(4):e00063520. https://doi.org/10.1590/0102-311X00063520.

13. Helioterio MC, Lopes FQRS, Sousa CC, Souza FO, Pinho PS, Souza FNF, et al. COVID-19: por que a proteção da saúde dos trabalhadores e trabalhadoras da saúde é prioritária no combate à pandemia?. Trab Educ Saude. 2020;18(3): e00289121. https://doi.org/10.1590/1981-7746-sol00289.

14. Ministério da Saúde (BR). Especial: doença pelo coronavírus COVID-19. Brasília, DF: MS; 2020. (Boletim Epidemiológico, n. 21).

15. Ruiu ML. Mismanagement of Covid-19: lessons learned from Italy. J Risk Res. 2020;23(78):1007-20. https://doi.org/10.1080/13669877.2020.1758755.

16. Areosa J, Queiros C. Burnout: uma patologia social reconfigurada na era COVID-19? Int J Work Cond. 2020;20:71-90. https://doi.org/10.25762/abh3-qh73.

17. Balog-Way DHP, McComas KA. COVID-19: reflections on trust, tradeoffs, and preparedness. J Risk Res. 2020;23(7-8):838-48. https://doi.org/10.1080/13669877.2020.1758192.

18. Salvetti MG, Pimenta CAM. Chronic pain and the belief in self-efficacy. Rev Esc Enferm USP. 2007;41(1):135-40. https://doi.org/10.1590/s0080-62342007000100018.

19. Ferreira MJ, Irigoyen MC, Consolim-Colombo F, Saraiva JFK, De Angelis K. Physically active lifestyle as an approach to confronting COVID-19. Arq Bras Cardiol. 2020;114(4):601-2. https://doi.org/10.36660/abc.20200235.

20. Stein R. Exercício físico em pacientes cardiopatas e na população em tempos de Coronavírus. Arq Bras Cardiol. 2020;114(5):827-8. https://doi.org/10.36660/abc.20200281.

21. Raiol RA. Praticar exercícios físicos é fundamental para a saúde física e mental durante a Pandemia da COVID-19. Braz J Heath Rev. 2020;3(2):2804-13. https://doi.org/10.34119/bjhrv3n2-124.

22. Andrade GC, Gombi-Vaca MF, Louzada MLC, Azeredo CM, Levy RB. The consumption of ultra-processed foods according to eating out occasions. Public Health Nutr. 2020;23(6):1041-8. https://doi.org/10.1017/S1368980019002623. 
23. Steele EM, Rauber F, Costa CS, Leite MA, Gabe KT, Louzada MLC, et al. Dietary in the NutriNet Brazil cohort during the COVID-19 pandemic. Rev Saude Publica. 2020 Sep 4;54:91. https://doi.org/10.11606/s1518-8787.2020054002950.

24. Fernandes LS, Nitsche MJT, Godoy I. Associação entre síndrome de burnout, uso prejudicial de álcool e tabagismo na enfermagem nas UTIs de um hospital universitário. Cienc Saude Colet. 2018;23(1):203-14. https://doi.org/10.1590/141381232018231.05612015.

25. Huh J, Timberlake DS. Do smokers of specialty and conventional cigarettes differ in their dependence on nicotine? Addict Behav. 2009;34(2):204-11. https://doi.org/10.1016/j.addbeh.2008.10.014

26. Lanctot JQ, Stockton MB, Mzayek F, Read M, McDevitt-Murphy M, Kenneth W. Effects of disasters on smoking and relapse: an exploratory study of Hurricane Katrina victims. Am J Healh Educ. 2008;39(2):91-4. https://doi.org/10.1080/19325037.2008.10599020.

27. Heath C, Sommerfield A, von Ungern-Sternberg BS. Resilience strategies to manage psychological distress amongst healthcare workers during the COVID-19 pandemic: a narrative review. Anaesthesia. 2020;75(10)1364-71. https://doi.org/10.1111/anae.15180.

28. Silva SM, Borges E, Abreu M, Queirós C, Baptista P, Felli V. Relação entre resiliência e burnout: Promoção da saúde mental e ocupacional dos enfermeiros. Rev Port Enferm Saude Ment. 2016;(16):41-8. http://dx.doi.org/10.19131/rpesm.0156. 


\section{Minicurrículo}

Alberto Sumiya | ORCiD: 0000-0003-0757-2186

Fisioterapeuta, Doutor em Ciências da Motricidade. Docente Adjunto do Curso de Medicina da Universidade Federal de Santa Catarina em Curitibanos, SC, Brasil.

Eloisa Pavesi | ORCiD: 0000-0003-0428-6694

Farmacêutica, Doutora em Farmacologia. Docente Adjunto do Curso de Medicina da Universidade Federal de Santa Catarina em Curitibanos, SC, Brasil.

Juliberta Alves de Macêdo | ORCiD: 0000-0002-8931-7391

Fisioterapeuta, Mestranda em Saúde Coletiva, Docente Auxiliar do Curso de Fisioterapia da Universidade Federal do Amazonas em Coari, AM, Brasil.

Gabriel Farhat | ORCiD: 0000-0002-1790-2540

Fisioterapeuta, Mestre em Ciências da Saúde, Ponta Grossa, PR, Brasil.

Celita Salmaso Trelha | ORCiD: 0000-0001-5643-9002

Fisioterapeuta, Doutora em Ciências da Saúde. Docente Associado do Curso de Fisioterapia da Universidade Estadual de Londrina, Londrina, PR, Brasil. 